\title{
Multi-resident activity recognition using multi-label classification in ambient sensing smart homes
}

\begin{abstract}
Activity recognition in smart home environment using wireless ambient sensing is a wellknown problem that is being researched very actively. Rapid development in the sensing technologies has made human activity recognition very important for various fields such as health care, home monitoring, surveillance, etc. In this paper, we describe the use of Classifier Chain method of the Multi-Label Classification approach to tackle the task of multi-resident activity recognition. We evaluate the developed model of Classifier Chain with K-Nearest Neighbor as base classifier on real world ARAS dataset which consists of two smart homes with evaluation metrics such as accuracy, precision and hamming loss. Through results, it can be inferred that Classifier Chain method successfully caters the problem of multi-resident activity recognition taking into consideration underlying label dependencies.
\end{abstract}

Keyword: Complex activity recognition; Smart home environment; Classifier chain; Multilabel classification; Wireless sensor network 